\title{
Anesthesia for Pulmonary Artery Bending and Patent Ductus Arteriosus Ligation with Miller-Dieker Syndrome Infant
}

\author{
Ki-jae Lee ${ }^{1}$, Keun Hyeong Lee ${ }^{1}$, Ye Sull Kim¹, Taewon Lee ${ }^{1}$, Deokkyu \\ Kim¹, Sang-Kyi Lee, Jeongwoo Lee ${ }^{1}$ and Jun Ho Lee ${ }^{1,2 *}$, \\ ${ }^{1}$ Department of Anesthesiology and Pain medicine, Chonbuk National University \\ Medical School and Hospital, South Korea \\ ${ }^{2}$ Research Institute of Clinical Medicine of Chonbuk National University, South Korea
}

\section{Case Report \\ Volume 4 Issue 4}

Received Date: October 16, 2019

Published Date: October 26, 2019

DOI: $10.23880 /$ accmj-16000162

*Corresponding author: Jun Ho Lee, MD, Department of Anesthesiology and Pain medicine, Chonbuk National University Medical School and Hospital, Geonji-ro 20, Deokjin-gu, Jeonju 54907, Korea, Tel: 82-63-250-1241, Fax:82-63250-1240; Email: gojuno@yahoo.com

\section{Abstract}

Miller-Dieker syndrome (MDS) is a rare disorder characterized by type I lissencephaly (smooth brain) and characteristic facial features including protruding foreheads, depressed temples, low-necked, protruding upper lip, and small jaws. Many MDS patients have congenital heart defect and growth retardation. If these MDS cases are accompanied by deformities such as VSD, palliative treatment may be needed to prolong life. There is a case report of general anesthesia in children with MDS undergoing laryngotracheal separation and percutaneous gastrostomy surgery. However, anesthetic management of correcting heart anomalies has not been reported. The authors faced an infant with MDS who requires emergent pulmonary artery bending and Patent ductus arteriosus (PDA) ligation, and performed general anesthesia. We describe a case of MDS patient who had correcting heart anomalies and review the anesthetic management of MDS.

Keywords: Miller-Dieker syndrome; Difficult airway; Congenital heart anomaly

Abbreviations: PDA: Patent Ductus Arteriosus; MDS: Miller-Dieker Syndrome; MAC: Minimum Alveolar Concentration (MAC); ICP: Intracranial Pressure.

\section{Background}

Miller-Dieker syndrome (MDS) is a rare neurodevelopmental genetic disease that occurs in childhood. MDS was firstly reported by Miller in 1963, and was found to be related to lissencephaly by Dieker in 1984 [1,2]. The syndrome is caused by gene deletion from a chromosomal mutation on 17p13.3. MDS is characterized by classic type I lissencephaly, microcephaly and distinctive facial appearance, and it commonly accompanies congenital heart defects and growth retardation [3]. The brain anomalies of MDS are caused by stopping the movement of nerve cells to the 


\section{Anaesthesia \& Critical Care Medicine Journal}

cerebral cortex at the time of 10-14 weeks of embryonic development, and they cause various neurological symptoms besides mental retardation and seizure. Typical facial appearances include prominent forehead, bitemporal hollowing, short nose with upturned nares low-necked, protruding upper lip, and small jaws [4]. If MDS is accompanied with cardiac deformities such as VSD, palliative treatment is required to prolong life. This report deals with a case of anesthetic management of correcting heart anomalies that has not been previously reported. Here, we present a case of MDS infant who had emergency pulmonary artery bending and patent ductus arteriosus ligation, and review the anesthetic management of MDS.

\section{Case Report}

A 2-month-old boy weighing $3.5 \mathrm{~kg}$ was referred for emergent pulmonary artery banding and patent ductus arteriosus (PDA) ligation under general anesthesia. Prenatal examination included a fetal neurosonography and echocardiogram that demonstrated lissencephaly and ventriculoseptal defect (VSD). The delivery had been normal after gestation of 40 weeks and 2 days. The weight at birth was 2,460g, and Apgar scores at birth were 9 and 10 at 1 and 5minute, respectively. After birth the baby was hypotonic and postnatal brain ultrasound presented findings of agyria. MRI showed that sulcus and fissure in the brain were not found, and a cortex was thickened to maximum of $17 \mathrm{~mm}$ (Figure 1). The findings were reasonable to diagnose classical lissencephaly type 1 . The infant also showed typical facial features of MDS such as flat nose, slightly upturned nares, small jaws and long distance between eyes. Also his ears were slightly below the normal level and his forehead was wider than normal (Figure 2).

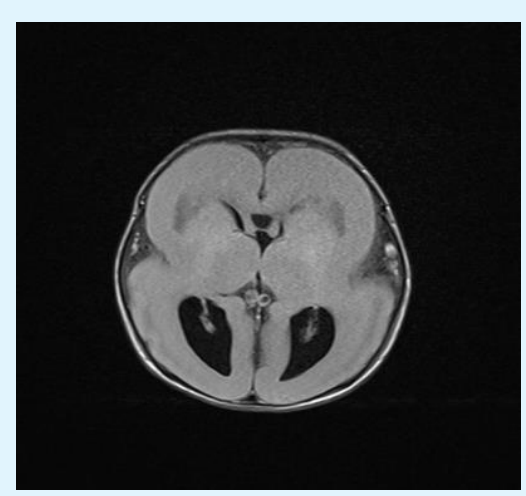

Figure 1: Magnetic Resonance Image (MRI) that suggest lissencephaly.

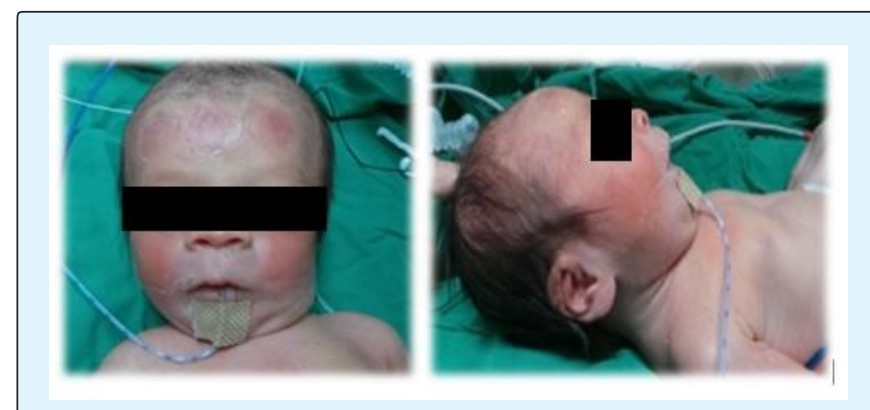

Figure 2: The infant that presents facial features of Miller-Dieker Syndrome.

Echocardiogram demonstrated a moderate perimembranous type of VSD at birth. Intermittent seizure like activity was observed, and epileptic encephalopathy was found in electroencephalography. After administering pentobarbital, seizure activity was not seen. Taken together, the authors diagnosed MDS although genetic test was not performed. After two months, the defect size of VSD was increased to $8 \mathrm{~mm}$. Also, desaturation up to $80 \%$, tachypnea and subcostal retraction were observed and aggravated. Although VSD closure might not be helpful, palliative pulmonary artery banding was decided to relieve symptoms above.

In pre-anesthetic examination, mild anemia with hemoglobin of $10.4 \mathrm{~g} / \mathrm{dl}$ was presented. Liver function tests were mildly increased, but serum electrolytes, blood sugar, coagulation profiles and electrocardiogram (ECG) was within normal range. Chest X-ray showed cardiomegaly and mild pneumonia. When arrived at the operating room, cardiac murmur and coarse breathing sound were heard on auscultation. ECG, pulse oximetry and Bispectral index (BIS; Medtronic, Minneapolis, USA) were monitored. The heart rate was 138 beats/min, blood pressure was $90 / 30 \mathrm{mmHg}$, oxygen saturation was $92 \%$, respiratory rate was 35 breaths/min, and BIS was less than 30. In case of difficult intubation, equipment for difficult airway such as bronchoscope and laryngeal mask airway were prepared, and three experienced anesthesiologists were ready.

After preoxygenation, general anesthesia was induced with thiopental sodium $10 \mathrm{mg}$ and rocuronium $4 \mathrm{mg}$, and sevoflurane was used to maintain anesthesia. With 3 minutes of mask ventilation, endotracheal intubation was attempted but failed. Laryngoscopic grade was IV that the laryngoscopic view was very limited. With the help of a bronchoscope, the authors successfully intubate a \# 3 endotracheal tube with a cuff, and the cuff pressure was 


\section{Anaesthesia \& Critical Care Medicine Journal}

monitored by a manometer. The $22 \mathrm{G}$ arterial line catheter was placed in the femoral artery, and the invasive arterial blood pressure was monitored.

During the operation, BIS was measured as 15-22 at end tidal sevoflurane concentration $1.4 \sim 2$ vol\%, which corresponded with $0.6 \sim 0.8$ minimum alveolar concentration (MAC). To prevent oxygen toxicity FiO2 was maintained to 0.4 , and oxygen saturation was maintained between 92 and 97\%. Mean arterial pressure was maintained between 40 and 60 during the entire operation. To achieve the hemodynamic stability dobutamine $5 \mathrm{mcg} / \mathrm{kg} / \mathrm{min}$ and fentanyl $8 \mathrm{mcg} / \mathrm{h}$ were continuously infused. After pulmonary artery banding with $4 \mathrm{~mm}$ Gore-Tex graft, pulmonary artery pressure was decreased from $30 / 23 / 14 \mathrm{mmHg}$ to $28 / 17 / 8 \mathrm{mmHg}$, and aorta pressure was changed from $44 / 34 / 40 \mathrm{mmHg}$ to 56/35/45 mmHg. Also, simple PDA ligation was completed safely. After the uneventful operation, mechanical ventilation was maintained without extubation and transferred to the pediatric intensive care unit. The patient was discharged with prescribed antiepileptic drug 16 days after the operation.

\section{Discussion}

MDS is a genetic disorder from gene depletion of chromosome $17 \mathrm{p} 13.3$, and is characterized by classical lissencephaly and distinct feature [5]. In this case, the patient presented typical craniofacial signs of MDS such as agyria in ultrasound and no sulcus and fissure in MRI. Also cortex of the brain was thickened that is corresponded to the typical characteristics of MDS. Typical features of MDS such as flat nose, slightly upturned nares and small jaws which were shown in our case. Because of the characteristics of MDS mentioned above, special care should be taken to manage anesthesia considering following factors. First, airway management can be challenging due to the typical facial features especially micrognathia. Preparation for difficult airway should be required before anesthesia. Second, BIS value of MDS patients is generally lower than normal, so that it is difficult to maintain adequate depth of anesthesia. Third, epileptic encephalopathies are common in MDS patients. Proper seizure control should be taken to avoid epilepsy during the operation.

Airway management cannot be overemphasized because of physical features of MDS patient. We must keep in mind the failure of intubation due to difficult airway, and preparation should be required before induction of anesthesia. In this case, three well-trained expert anesthesiologists and additional method of securing airway such as fiberoptic and videolaryngoscopy were ready. Also, sugammadex was ready for use at any time just in case. The patient had short neck, small jaw and mandible so that difficult airway can be anticipated. Also, the infant with MDS previously had repeated pneumonia and possible lung damage. After the failure of intubation with conventional method, fiberoptic intubation was attempted and airway was successfully secured.

BIS values were measured at 15-22 during the anesthetic course. Some case reports indicated that children with lissencephaly presented low BIS values like our case although BIS values are less reliable in children less than 2 years old [4,6]. Because BIS was lower than normal range, it is difficult to maintain the adequate depth of anesthesia. In order to provide adequate anesthesia, the authors used inhalation anesthetics instead of intravenous anesthetics or opioid. In addition, dobutamine and fentanyl were used to maintain proper vital signs, and sevoflurane was kept below 1 MAC.

Seizures occur in more than $90 \%$ of infant with lissencephaly. Most of them have infantile spasm, but EEG is usually nonspecific [4]. However, our case presented epileptic encephalopathy on EEG patterns. Ventricular dilatation, agyria, muscular hypotonia, or thickened cortex would be presented in this patient. This would be a factor of increasing intracranial pressure (ICP). In our case, the infant presented intermittent seizure, but we controlled it with pentobarbital and no further epilepsy was aggravated before and after anesthesia. Also, one of important goal of general anesthesia is to keep ICP within the normal range. The authors tried to keep adequate mean arterial pressure during the operation. Intraoperative control of seizure is also important during anesthesia. Our patient had a couple of vomiting episodes before anesthesia but no other signs of increased ICP such as unconsciousness, hypertension, dysarrhythmia and seizures was presented after surgery.

MDS may accompany with cardiac malformations, and death usually occurs in the first few years of life [3]. The cardiac findings of VSD and pulmonic stenosis are not typical of MSD, accounting for less than 10\% [7]. While the treatment of MDS with cardiac anomalies is not usually available, palliative and supportive treatment can be done only if diversity of the symptoms is presented. The infant in our case had symptoms of desaturation and 


\section{Anaesthesia \& Critical Care Medicine Journal}

subcostal retraction for breathing. Also, the size of VSD and shunt flow was increased. Hence, pulmonary artery bending was conducted to reduce shunt, but VSD closure has not been conducted considering that the remaining life would be short. In our case pulmonary artery pressure was successfully decreased after surgery.

Because the hyperthermia risk is increased in patients with lissencephaly syndrome from genetic depletion, body temperature was monitored throughout the surgery and postoperative period as diagnostic criteria for Walker-Warburg syndrome, another autosomal genetic disorder that has lissencephaly [8]. MDS patients are at high risk of aspiration [4]. As our case repetitive pneumonia might be more likely to cause aspiration pneumonia. Anesthetic management should include keeping the airway clean from accumulation of secretions during the surgery and observing adequate fasting period. There is a limitation that MDS has not been confirmed due to the lack of chromosomal study, but our patient can be strongly suspicious of MDS by ultrasound, MRI and characteristic features. In accordance with the congenital genetic disorder, anesthetic management was performed to uneventfully recover after surgery.

In summary, correction of heart abnormalities is not common in MDS patients, but palliative pulmonary artery banding can be performed. Anesthetic consideration should be included to prepare for difficult airway and maintain adequate depth of anesthesia due to lower BIS value.

\section{References}

1. Miller JQ (1963) Lissencephaly in 2 siblings. Neurology 13: 841-850.
2. Stratton RF, Dobyns WB, Airhart SD, Ledbetter DH (1984) New chromosomal syndrome: Miller-Dieker syndrome and monosomy 17p13. Hum Genet 67(2): 193-200.

3. Atwal PS, Macmurdo C (2015) A Case of Concurrent Miller-Dieker Syndrome (17p13.3 Deletion) and 22q11.2 Deletion Syndrome. J Pediatr Genet 4(4): 201-203.

4. Wakiguchi C, Godai K, Mukaihara K, Ohnou T, Kuniyoshi T, et al. (2015) Management of general anesthesia in a child with Miller-Dieker syndrome: a case report. JA Clin Rep 1(1): 14.

5. Chabchoub I, Maalej B, Belguith N, Chaabouni M, Mansour LB, et al. (2011) Miller-Dieker syndrome: A report of a new case and review of the literature. J Pediatr Neurol 09(3): 387-390.

6. Valkenburg AJ, De Leeuw TG, Machotta A, Weber F (2008) Extremely low preanesthetic BIS values in two children with West syndrome and lissencephaly. Pediatr Anesth 18(5): 446-448.

7. Chen CP, Chang TY, Guo WY, Wu PC, Wang LK, et al. (2013) Chromosome 17p13.3 deletion syndrome: aCGH characterization, prenatal findings and diagnosis, and literature review. Gene 532(1): 152159.

8. Dobyns WB, Pagon RA, Armstrong D, Curry CJR, Greenberg F, et al. (1989) Diagnostic criteria for Walker-Warburg syndrome. American Journal of Medical Genetics 32(2): 195-210. 